\title{
A pretty binomial identity
}

Tewodros Amdeberhan, Valerio de Angelis, Minghua Lin, Victor H. Moll, B. Sury

Tewodros Amdeberhan obtained his Ph.D. in mathematics from Temple University. He currently teaches at Tulane University and holds a permanent membership at DIMACS, Rutgers University.

Valerio de Angelis obtained his Ph.D. in mathematics from the University of Washington in Seattle. He is a professor of mathematics at Xavier University in Louisiana. Minghua Lin is currently a graduate student in mathematics at the University of Regina.

Victor H. Moll obtained his Ph.D. in mathematics from the Courant Institute of New York University. He is a professor of mathematics at Tulane University.

B. Sury obtained his Ph.D. in mathematics from the Tata Institute of Fundamental Research in Mumbai. He is a professor of mathematics at the Indian Statistical Institute in Bangalore.

\section{Introduction}

The evaluation of finite sums involving binomial coefficients appears throughout the undergraduate curriculum. Students are often exposed to identities such as

$$
\sum_{k=0}^{n}\left(\begin{array}{l}
n \\
k
\end{array}\right)=2^{n} \quad \text { and } \quad \sum_{k=0}^{n}\left(\begin{array}{l}
n \\
k
\end{array}\right)^{2}=\left(\begin{array}{c}
2 n \\
n
\end{array}\right) .
$$

Identitäten, die Binomialkoeffizienten beinhalten, tauchen in der mathematischen Literatur in den verschiedensten Bereichen auf. Ein Beispiel dafür ist die uns aus der Schule bekannte Formel $\sum_{k=0}^{n}\left(\begin{array}{l}n \\ k\end{array}\right)=2^{n}$, welche sich unmittelbar aus dem binomischen Lehrsatz ergibt. In der vorliegenden Arbeit untersuchen die Autoren eine Variation dieses Themas und beweisen eine geschlossene Formel für eine Summe von Produkten von Binomialkoeffizienten. Interessant daran ist, dass vier verschiedene Beweise für diese Formel gegeben werden: ein erster Beweis mit Hilfe der Methode von Wilf/Zeilberger, ein zweiter unter Verwendung von Laurent-Polynomen, ein dritter mit hypergeometrischen Funktionen und ein letzter Beweis, der komplexe Integration verwendet. Die Arbeit schließt mit einem noch unvollständigen kombinatorischen Beweis, der dem Leser als Übungsaufgabe überlassen ist. 
Elementary proofs abound: the first identity results from choosing $x=y=1$ in the binomial expansion of $(x+y)^{n}$. The second one may be obtained by comparing the coefficient of $x^{n}$ in the identity $(1+x)^{n}(1+x)^{n}=(1+x)^{2 n}$. The reader is surely aware of many other proofs, including some combinatorial in nature.

At the end of the previous century, the evaluation of these sums was trivialized by the work of H. Wilf and D. Zeilberger [8]. In the preface to the charming book, the authors begin with the phrase

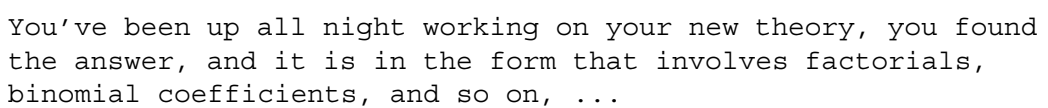

and then proceed to introduce the method of creative telescoping discussed in Section 3. This technique provides an automatic tool for the verification of these type of identities. The points of view presented in [3] and [10] provide an entertaining comparison of what is admissible as a proof.

In this short note we present a variety of proofs of the identity

$$
\sum_{k=0}^{m} 2^{-2 k}\left(\begin{array}{c}
2 k \\
k
\end{array}\right)\left(\begin{array}{c}
2 m-k \\
m
\end{array}\right)=\sum_{k=0}^{m} 2^{-2 k}\left(\begin{array}{c}
2 k \\
k
\end{array}\right)\left(\begin{array}{c}
2 m+1 \\
2 k
\end{array}\right) .
$$

The common value is

$$
2^{-2 m}\left(\begin{array}{c}
4 m+1 \\
2 m
\end{array}\right)=2^{-2 m-1}\left(\begin{array}{c}
4 m+2 \\
2 m+1
\end{array}\right)
$$

\section{The origin}

The formula (1.2) comes from an unexpected source. Several evaluations of

$$
N_{0,4}(a ; m):=\int_{0}^{\infty} \frac{d x}{\left(x^{4}+2 a x^{2}+1\right)^{m+1}},
$$

for $a>-1$ and $m \in \mathbb{N}$, in the form

$$
N_{0,4}(a ; m)=\frac{\pi}{2^{m+3 / 2}(a+1)^{m+1 / 2}} P_{m}(a),
$$

are given in [1]. Here $P_{m}(a)$ is a polynomial in $a$. The first expression obtained for $P_{m}(a)$ in [6], via elementary methods, is

$$
P_{m}(a)=\sum_{j=0}^{m}\left(\begin{array}{c}
2 m+1 \\
2 j
\end{array}\right)(a+1)^{j} \sum_{k=0}^{m-j}\left(\begin{array}{c}
m-j \\
k
\end{array}\right)\left(\begin{array}{c}
2(m-k) \\
m-k
\end{array}\right) 2^{-3(m-k)}(a-1)^{m-k-j} .
$$

The reader will find the details in [5, p. 140]. The alternative expression

$$
P_{m}(a)=2^{-2 m} \sum_{k=0}^{m} 2^{k}\left(\begin{array}{c}
2 m-2 k \\
m-k
\end{array}\right)\left(\begin{array}{c}
m+k \\
m
\end{array}\right)(a+1)^{k}
$$

appeared first in [4]. The complexity of these expressions lead the authors to evaluate it at $a=1$. This produced (1.2). 


\section{A proof using the $\mathrm{WZ}$ method}

An efficient procedure to prove the identity $a_{n}=b_{n}$ is to produce a recurrence satisfied by both sequences and matching the required initial conditions. For example, to prove

$$
\sum_{k=0}^{n}\left(\begin{array}{l}
n \\
k
\end{array}\right)=2^{n},
$$

it suffices to check that both sides satisfy $x_{n}=2 x_{n-1}$ and they share the initial condition $x_{0}=1$. The real question is how to produce the recurrence. In this case, it is easier to look for a recurrence for the summand $\left(\begin{array}{l}n \\ k\end{array}\right)$ and then sum over $k$. The basic identity

$$
\left(\begin{array}{l}
n \\
k
\end{array}\right)=\left(\begin{array}{c}
n-1 \\
k
\end{array}\right)+\left(\begin{array}{l}
n-1 \\
k-1
\end{array}\right)
$$

after summing over all values of $k$ yields the desired recurrence. The result now follows from checking initial values.

H. Wilf and D. Zeilberger [7, 8, 9] came up with a breakthrough in producing an algorithm that provides the recurrence for the summand $t_{n, k}$ in $s_{n}=\sum_{k} t_{n, k}$. The method applies to terms $t_{n, k}$ of hypergeometric type; that is $t_{n+1, k} / t_{n, k}$ and $t_{n, k+1} / t_{n, k}$ are rational functions of the indices. This so-called WZ-method has been implemented in modern symbolic languages. For instance, Maple shows that both sides of (1.2) satisfy the recurrence

$$
(2 m+3)(2 m+2) f(m+1)=(4 m+5)(4 m+3) f(m) .
$$

The identity (1.2) now follows from the fact that both sides reduce to 1 at $m=0$. Furthermore, iterating (3.1) yields

$$
f(m)=2^{-2 m}\left(\begin{array}{c}
4 m+1 \\
2 m
\end{array}\right)
$$

\section{A constant term approach}

The second identity in (1.1) arises from matching the coefficient of $x^{n}$ in the trivial identity $(1+x)^{n}(1+x)^{n}=(1+x)^{2 n}$. The proof of (1.2) presented in this section is based on producing (Laurent) polynomials whose constant terms give the two sides, respectively. The presence of the binomial coefficient $\left(\begin{array}{c}2 m+1 \\ 2 k\end{array}\right)$ in (1.2) suggests to consider

$$
\begin{aligned}
\left(1+\frac{t+t^{-1}}{2}\right)^{2 m+1} & =\sum_{r=0}^{2 m+1} 2^{-r}\left(\begin{array}{c}
2 m+1 \\
r
\end{array}\right)\left(t+t^{-1}\right)^{r} \\
& =\sum_{r=0}^{2 m+1} 2^{-r} \sum_{k=0}^{r}\left(\begin{array}{c}
2 m+1 \\
r
\end{array}\right)\left(\begin{array}{l}
r \\
k
\end{array}\right) t^{k} t^{-(r-k)} .
\end{aligned}
$$

Its constant term is

$$
\sum_{k=0}^{m} 2^{-2 k}\left(\begin{array}{c}
2 k \\
k
\end{array}\right)\left(\begin{array}{c}
2 m+1 \\
2 k
\end{array}\right)
$$


which is the right-hand side of (1.2). From

$$
\left(1+\frac{t+t^{-1}}{2}\right)^{2 m+1}=\frac{(t+1)^{4 m+2}}{(2 t)^{2 m+1}}
$$

this constant term evaluates to $2^{-(2 m+1)}\left(\begin{array}{c}4 m+2 \\ 2 m+1\end{array}\right)$.

The left-hand side comes from the constant term in the identity $(1+t)^{-1 / 2} \times(1+t)^{-m-1}=$ $(1+t)^{-m-3 / 2}$. In this product, employ the expansions

$$
(1+t)^{-1 / 2}=\sum_{k=0}^{\infty} 2^{-2 k}\left(\begin{array}{c}
2 k \\
k
\end{array}\right)(-t)^{k} \text { and }(1+t)^{-m-1}=\sum_{k=0}^{\infty}\left(\begin{array}{c}
m+k \\
k
\end{array}\right)(-t)^{k}
$$

to compute the coefficient of $t^{m}$ of the product. Observe that only terms up to order $m$ contribute to this computation. Thus,

$$
\sum_{k=0}^{m}\left(\begin{array}{c}
2 m-k \\
m
\end{array}\right)(-t)^{m-k}=\sum_{k=0}^{m}\left(\begin{array}{c}
2 m-k \\
m-k
\end{array}\right)(-t)^{k}
$$

shows that the constant term of $t^{-m}(1+t)^{-m-1}(1+t)^{-1 / 2}$ is the left-hand side of (1.2). That is,

$$
(-1)^{m}\left(\begin{array}{c}
-m-\frac{3}{2} \\
m
\end{array}\right)=\frac{1}{2^{2 m+1}}\left(\begin{array}{l}
4 m+2 \\
2 m+1
\end{array}\right)
$$

giving the result.

\section{An excursion into the hypergeometric world}

A natural setting of binomial sums such as (1.2) is in the context of hypergeometric functions. These are functions defined by a power series $\sum_{n=0}^{\infty} a_{n} x^{n}$ where the ratio $a_{n+1} / a_{n}$ is a rational function of the index $n$. A classical example is given by

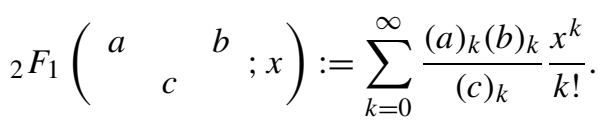

Here $(a)_{k}=a(a+1)(a+2) \ldots(a+k-1)$ is the ascending factorial. Observe that the function ${ }_{2} F_{1}$ is symmetric in $a$ and $b$. Moreover, if $a$ is a negative integer, the sum is finite and the function reduces to a polynomial.

Proposition 5.1 The identity (1.2) is equivalent to

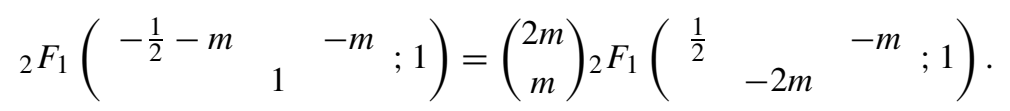


Proof. The relations $\left(\frac{1}{2}\right)_{k}=2^{-2 k}(2 k) ! / k$ ! and

$$
(-r)_{k}= \begin{cases}\frac{(-1)^{k} r !}{(r-k) !} & \text { for } 0 \leq k \leq r, \\ 0 & \text { otherwise, }\end{cases}
$$

show that the right-hand side of (1.2) and (5.1) agree. Similarly, the relation

$$
\left(-\frac{1}{2}-m\right)_{k}=\frac{(-1)^{k}(2 m+1) !(m-k) !}{2^{2 k}(2 m-2 k+1) ! m !}
$$

shows that the left-hand side of (1.2) and (5.1) agree.

The hypergeometric terms in (5.1) can be evaluated using a classical formula of Gauss [2]:

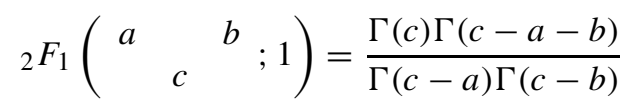

and the specialization

$$
{ }_{2} F_{1}\left(\begin{array}{ccc}
-n & & b \\
& c & ; 1
\end{array}\right)=\frac{(c-b)_{n}}{(c)_{n}} .
$$

The identity

$$
(-x)_{n}=(-1)^{n}(x-n+1)_{n}
$$

completes the argument.

\section{A contour integration approach}

Complex analytic techniques are useful in establishing identities involving binomial coefficients. The representation

$$
\left(\begin{array}{c}
m \\
n
\end{array}\right)=\frac{1}{2 \pi i} \int_{|z|=r} \frac{(1+z)^{m}}{z^{n+1}} d z
$$

for $r>0$ provides the relevant connection. This will now be employed to produce a proof of (1.2).

The left-hand side becomes, for an appropriate choice of radii $r_{1}$ and $r_{2}$,

$$
\begin{aligned}
\sum_{k=0}^{\infty} 2^{-2 k}\left(\begin{array}{c}
2 k \\
k
\end{array}\right)\left(\begin{array}{c}
2 m+1 \\
2 k
\end{array}\right) & =\frac{1}{(2 \pi i)^{2}} \sum_{k \geq 0} 2^{-2 k} \int_{|z|=r_{1}} \frac{(1+z)^{2 k}}{z^{k+1}} d z \int_{|w|=r_{2}} \frac{(1+w)^{2 m+1}}{w^{2 k+1}} d w \\
& =\frac{1}{(2 \pi i)^{2}} \int_{|z|=r_{1}} \int_{|w|=r_{2}} \frac{(1+w)^{2 m+1}}{z w} \sum_{k=0}^{\infty}\left[\frac{(1+z)^{2}}{4 z w^{2}}\right]^{k} d w d z \\
& =\frac{1}{2 \pi i} \int_{|z|=r_{1}} \frac{1}{2 \pi i} \int_{|w|=r_{2}} \frac{4 w(1+w)^{2 m+1}}{4 z w^{2}-(1+z)^{2}} d w d z .
\end{aligned}
$$


The choice of $r_{2}=1$ and Cauchy's residue theorem imply that the inner integral is the sum of the residues at the poles $w= \pm(1+z) / 2 \sqrt{z}$. This yields

$$
\begin{aligned}
& \sum_{k=0}^{\infty} 2^{-2 k}\left(\begin{array}{c}
2 k \\
k
\end{array}\right)\left(\begin{array}{c}
2 m+1 \\
2 k
\end{array}\right) \\
& \quad=\frac{1}{2 \pi i} \int_{|z|=r_{1}} \frac{1}{2^{2 m+2} z^{m+3 / 2}}\left[\left(1+z^{1 / 2}\right)^{4 m+2}-\left(1-z^{1 / 2}\right)^{4 m+2}\right] d z
\end{aligned}
$$

Expand the integrand to verify that it is a rational function of $z$ which is analytic away from the origin. The pole at $z=0$ is of order $m+1$ with residue $2^{-2 m-1}\left(\begin{array}{l}4 m+2 \\ 2 m+1\end{array}\right)$. This produces the right-hand side of (1.2).

The left-hand side of (1.2) is

$$
\begin{aligned}
\sum_{k \geq 0} 2^{-2 k}\left(\begin{array}{c}
2 k \\
k
\end{array}\right)\left(\begin{array}{c}
2 m-k \\
m-k
\end{array}\right) & =\frac{1}{(2 \pi i)^{2}} \sum_{k \geq 0} 2^{-2 k} \int_{|z|=\frac{1}{2}} \frac{(1+z)^{2 k}}{z^{k+1}} d z \int_{|w|=\frac{1}{5}} \frac{(1+w)^{2 m-k}}{w^{m-k+1}} d w \\
& =\frac{1}{(2 \pi i)^{2}} \int \frac{(1+w)^{2 m}}{w^{m+1}} \int \frac{1}{z} \sum_{k \geq 0}\left(\frac{w(1+z)^{2}}{4 z(1+w)}\right)^{k} d z d w \\
& =\frac{1}{(2 \pi i)^{2}} \int \frac{1}{2} \frac{(1+w)^{2 m+1}}{w^{m+1}} \int \frac{4 d z}{|w|=\frac{1}{5}} d z(1+w)-w(1+z)^{2}
\end{aligned}
$$

The only pole of the integrand, inside the contour, is $z=\frac{1}{w}(2+w-2 \sqrt{1+w})$. Thus

$$
\frac{1}{2 \pi i} \int_{|z|=\frac{1}{2}} \frac{4 d z}{-w z^{2}+(4+2 w) z-w}=\frac{1}{\sqrt{1+w}} .
$$

This shows that the left-hand side is

$$
\frac{1}{2 \pi i} \int_{|w|=\frac{1}{5}} \frac{(1+w)^{2 m+\frac{1}{2}}}{w^{m+1}}=\left(\begin{array}{c}
2 m+\frac{1}{2} \\
m
\end{array}\right) .
$$

The identity

$$
\left(\begin{array}{c}
2 m+\frac{1}{2} \\
m
\end{array}\right)=2^{-2 m}\left(\begin{array}{c}
4 m+1 \\
2 m
\end{array}\right)
$$

completes the proof. 


\section{A partial combinatorial proof}

Consider the set $X$ of lattice paths in the plane that start at $(0,0)$, taking unit steps $N=$ $(0,1), S=(0,-1), E=(1,0)$ and $W=(-1,0)$, of odd length $2 m+1$, and ending at the $y$-axis. It is clear that the number of $E$ steps is the same as the $W$ steps, call it $j$. Choose the steps that are either $E$ or $W$ in $\left(\begin{array}{c}2 m+1 \\ 2 j\end{array}\right)$ ways. Then choose which is $E$ and which is $W$ in $\left(\begin{array}{c}2 j \\ j\end{array}\right)$ ways. Finally choose the remaining $2 m+1-2 j$ steps to be either $N$ or $S$ in $2^{2 m+1-2 j}$ ways. This gives

$$
|X|=\sum_{j=0}^{m}\left(\begin{array}{c}
2 m+1 \\
2 j
\end{array}\right)\left(\begin{array}{c}
2 j \\
j
\end{array}\right) 2^{2 m+1-2 j} .
$$

Thus, the right-hand side of (1.2) gives the cardinality of the set $X$, aside from the factor $2^{2 m+1}$.

The size of $X$ is now evaluated by exhibiting a bijection between $X$ and $Y$ that makes counting simpler. The new set $Y$ is formed by all paths on the $x$-axis that start and end at 0 , take steps $e=+1$ and $w=-1$, and have length $4 m+2$. It will be shown that there is a bijection from $Y$ to $X$. Clearly there must be $2 m+1$ of each kind of steps, and so the size of $Y$ is

$$
|Y|=\left(\begin{array}{l}
4 m+2 \\
2 m+1
\end{array}\right)
$$

The proof is completed by noticing that there is a simple bijection between $X$ and $Y$ given by

$$
E \mapsto e e, \quad W \mapsto w w, \quad N \mapsto e w, \quad S \mapsto w e .
$$

It remains to produce a combinatorial interpretation of the left-hand side of (1.2). The reader is invited to produce one: the authors have been unable to do so.

\section{Acknowledgement}

The work of the fourth author was partially supported by NSF-DMS 0070567.

\section{References}

[1] Amdeberhan, T.; Moll, V.: A formula for a quartic integral: a survey of old proofs and some new ones. Ramanujan J. 18 (2009), 91-102.

[2] Andrews, G.E.; Askey, R.; Roy, R.: Special Functions. Vol. 71, Encyclopedia of Mathematics and its Applications. Cambridge Univ. Press, New York 1999.

[3] Andrews, G.E.: The death of proof? Semi-rigorous Mathematics? You've got to be kidding! Math. Intelligencer 16 (1994), 16-18.

[4] Boros, G.; Moll, V.: The double square root, Jacobi polynomials and Ramanujan's master theorem. $J$. Comput. Appl. Math. 130 (2001), 337-344.

[5] Boros, G.; Moll, V.: Irresistible Integrals. Cambridge Univ. Press, New York 2004.

[6] Boros, G.; Moll, V.; Riley, S.: An elementary evaluation of a quartic integral. Sci. Ser. A Math. Sci. (N.S.) 11 (2005), 1-12. 
[7] Nemes, I.; Petkovsek, M.; Wilf, H.S.; Zeilberger, D.: How to do MONTHLY problems with your computer. Amer. Math. Monthly 104 (1997), 505-519.

[8] Petkovsek, M.; Wilf, H.S.; Zeilberger, D.: A=B. A.K. Peters, Ltd., 1st edition, 1996.

[9] Wilf, H.S.; Zeilberger, D.: An algorithmic proof theory for hypergeometric (ordinary and " $q$ ") multisum/integral identities. Invent. Math. 108 (1992), 575-633.

[10] Zeilberger, D.: Theorems for a price: Tomorrow's semi-rigorous mathematical culture. Notices Amer. Math. Soc. 40 (1993), 978-981.

Tewodros Amdeberhan

Victor H. Moll

Department of Mathematics

Tulane University

New Orleans, LA 70118, USA

e-mail: tamdeber@tulane.edu

vhm@math.tulane.edu

Valerio de Angelis

Department of Mathematics

Xavier University of Louisiana

New Orleans, LA 70125, USA

e-mail: vdeangel@xula.edu

Minghua Lin

Department of Mathematics and Statistics

University of Regina

Saskatchewan, S4S 0A2, Canada

e-mail: lin243@math.uregina.ca

B. Sury

Statistics and Mathematics Unit

Indian Statistical Institute

8th Mile Mysore Road

Bangalore 560059, India

e-mail: sury@isibang.ac.in 